\title{
INNOVAR CON PERSPECTIVA DE GÉNERO EN LOS GRADOS DE CIENCIAS SOCIALES. LA EXPERIENCIA EN LA UNIVERSIDAD DE JAÉN
}

\section{INNOVATION WITH GENDER APPROACH ON SOCIAL SCIENCE DEGREES: THE EXPERIENCE IN THE UNIVERSITY OF JAÉN}

Belén Blázquez Vilaplana (Universidad de Jaén) ${ }^{1}$

Javier Alarcón González (Universidad de Jaén) ${ }^{2}$

\begin{abstract}
Resumen:
El trabajo parte de la reflexión realizada a partir de varios proyectos de innovación docente y de un curso de formación docente, ambos bajo la óptica de la perspectiva de género en la Universidad de Jaén. A partir de la contextualización de la perspectiva de género en la normativa legal y universitaria, se introduce el concepto y su aplicación a la docencia universitaria. Esto supone someter a reflexión y reorganización los contenidos, la metodología y actividades, el sistema de evaluación y las fuentes de referencia. Se establecen una serie de acciones docentes para incorporar la perspectiva de género con el objetivo de contribuir a cambiar las percepciones de los estudiantes y provocar una mayor concienciación y sensibilización a la igualdad.
\end{abstract}

Palabras claves: Perspectiva de género; docencia universitaria; Ciencias Sociales.

Códigos JEL: A22, A23, I23.

\begin{abstract}
:
This article focuses on the reflexion made from several university teaching innovation projects and from a teacher-training course, at the University of Jaén, both with a gender approach. Based on the contextualization of the gender perspective in legal and university regulations, the concept and its application to university teaching are introduced. This involves putting forward for discussion the contents, methodology and activities, evaluation system and reference sources. A series of teaching actions are established to incorporate the gender perspective with the objective of contributing to change the perceptions of students and raise awareness and sensitivity to the gender equality issue.
\end{abstract}

Key words: Gender approach; University teaching; Social Sciences.

JEL Codes: A22, A23, I23.

\section{INTRODUCCIÓN}

El objetivo de este trabajo es dar pautas para introducir la perspectiva de género en la docencia universitaria, concretamente en los grados de Ciencias Sociales a partir de las experiencias derivadas de distintas actuaciones en la Universidad de Jaén. Consideramos que en esta rama de conocimiento, es fundamental trabajar bajo esta metodología porque, la mayor parte de las temáticas que se tratan, y los resultados y las aplicaciones de los

\footnotetext{
${ }^{1}$ bblazquez@ujaen.es, Universidad de Jaén.

2 falarcon@ujan.es, Universidad de Jaén.

Recibido 23 de septiembre de 2019. Aceptado 27 de noviembre de 2019.
} 
contenidos, afectan directamente a las personas o indirectamente a través de las instituciones y organizaciones que regulan la interacción social (Agéncia per a la Qualitat del Sistema Universitari de Catalunya, 2019) ${ }^{3}$. Entendiendo que la perspectiva de género es un elemento de innovación si atendemos a lo que la Real Academia de la Lengua Española reconoce como la primera acepción de la palabra innovación: "mudar o alterar algo, introduciendo novedades” (RAE, s/f). Ello, porque aquellas personas que dentro de las Universidades trabajan - o lo intentan - con perspectiva de género, lo que buscan es cambiar las estructuras patriarcales y trasformar las prácticas educativas ${ }^{4}$. En palabras de Donoso (2018, p.35):

Requiere trasformar las conciencias de futuros profesionales y hacerlos permeables a las relaciones generizadas que van a encontrarse en los futuros puestos de trabajo. La elección de enseñar un conjunto de valores, ideas, suposiciones e informaciones, y, al hacerlo, omitir otros valores, otras ideas, otras suposiciones y otras informaciones, se convierte en un acto político.

La hipótesis con la que se ha venido trabajando es que a pesar de la existencia de un marco normativo nacional y autonómico que promueve la introducción de la perspectiva de género en la docencia universitaria, esta es residual y se ha dejado en manos de la voluntad del profesorado y de los equipos de gobierno de las distintas facultades y Universidades. En el mejor de los casos, se han introducido algunas asignaturas optativas en los grados y máster oficiales pero sin realizarse una revisión en profundidad de las competencias, contenidos, metodologías y sistemas de evaluación recogidas en las guías docentes. Como afirma la profesora Buquet (2011, p.215), “al no ser materias incorporadas en la estructura del plan de estudios, o sea, institucionalizadas, aparecen y desaparecen en función del interés del profesorado". Mientras la docencia con perspectiva de género no deje de ser sólo una posibilidad en el marco del Espacio Europeo de Educación Superior, sino que se acate como de obligado cumplimiento la normativa ya referida en todos sus niveles y sea tenida en cuenta a la hora, por ejemplo, de valorar la evaluación del programa DOCENTIA del profesorado, introducirla en el proceso de enseñanza-aprendizaje seguirá siendo una cuestión de orden menor tanto para la formación del profesorado, como para la transmisión del conocimiento al estudiantado. Y, por tanto, deberemos seguir considerando que quién la incorpore en su docencia estará innovando.

Para dar respuesta a las inquietudes y objetivos planteados, recogeremos las experiencias derivadas, en más de cuatro años, de varios proyectos de innovación docentes implementados en la Facultad de Ciencias Sociales y Jurídicas de la Universidad de Jaén desde el curso $2014^{5}$ y de los resultados del curso de Formación "Integrar la perspectiva de

\footnotetext{
${ }^{3}$ Además, es imposible obviar, si queremos trabajar con perspectiva de género, su inclusión en cualquier materia y/o asignatura sobre metodología y técnicas de investigación social.

${ }^{4}$ La profesora Donoso Vázquez se preguntaba cómo podría ser un motor de innovación la introducción de la perspectiva de género. Según afirmaba, no se le puede aplicar el concepto de innovación si lo que se plantea es poner sobre la mesa la necesidad de trabajar y aceptar la igualdad entre hombres y mujeres. "Ya que es una petición del ser humano desde la revolución francesa, a partir de la cual se instauraron todas las democracias modernas. Una petición tan antigua no podría tratarse de innovadora. Es cierto que en esa petición de igualdad no estaban incluidas las mujeres, a las cuales se las olvidó porque prácticamente eran inexistentes, no en número, sino en valor social. Esta conceptualización pertenece a mí entender a un primer nivel de la perspectiva de género, aquella que pretende incidir en las desigualdades de género existentes para nivelarlas” (Donoso, 2018, p. 24). De ahí que lo que plantee calificar como innovación sea un segundo nivel que lo que busque no sea introducir ajustes, sino cambiar las estructuras.

${ }^{5}$ Proyecto PID10_201416, “Competencias en género e igualdad de oportunidades: indicadores y herramientas de evaluación”, de la Convocatoria 2014/16 del Vicerrectorado de Docencia y Profesorado de la Universidad de Jaén; y, del proyecto PID 73_201617, “Análisis descriptivo de la perspectiva de género en los grados impartidos en la Facultad de Ciencias Sociales y Jurídicas“, de la convocatoria 2016/18 del Vicerrectorado de Enseñanza de Grado, Postgrado y Formación Permanente de la Universidad de Jaén.
} 
género en la docencia universitaria. Protocolo de buenas prácticas” impartido al Personal Docente e Investigador durante el curso 2018/19 a través del Vicerrectorado de Ordenación Académica y Profesorado. De esta forma también queremos dar a conocer esta línea de trabajo, que se ha venido implementando en la facultad de Ciencias Sociales y Jurídicas de la Universidad de Jaén: la docencia con perspectiva de género y sus aportaciones a la innovación.

Una vez plateados nuestro objetivo y la justificación, el presente artículo se estructura de la siguiente manera. El siguiente apartado se contextualiza la normativa tanto en el ámbito de igualdad como universitario que reconoce la necesidad de intervención. A continuación se conceptualiza la perspectiva de género en el contexto universitario con especial incidencia en el ámbito docente. Una vez contextualizado y definida la perspectiva de género el texto esclarece las pautas para la introducción de la perspectiva de género en la estructura organizativa de la Universidad, en las guías docentes y en el curriculum formal y en el oculto. En este mismo apartado se profundiza en las acciones a realizar para introducir dicha perspectiva en los contenidos, en la metodología y acciones, en el sistema de evaluación y en la bibliografía. El articulo, en último lugar, establece unas conclusiones.

\section{CONTEXTUALIZACIÓN DE LA NORMATIVA EN EL ÁMBITO DE LA IGUALDAD Y EN EL UNIVERSITARIO}

Para poder analizar cómo introducir la perspectiva de género en los estudios universitarios es necesario hacer una breve referencia al contexto del Espacio Europeo de Educación Superior y a la Ley Orgánica de Igualdad Efectiva entre Mujeres y Hombres. Este será el marco necesario para entender los retos y los desafíos a los que se enfrenta la Universidad si su fin es convertirse en un espacio de docencia e investigación no androcéntrico.

El 19 de junio de 1999, 29 Estados europeos, entre los que se encontraba España, firmaron lo que se conocería como la Declaración de Bolonia ${ }^{6}$, la cual sentaría las bases para la consecución de un Espacio Europeo de Educación Superior. Lo que en un principio fue visto como el impulso necesario para adaptar las Universidades del viejo continente a los procesos de globalización y a la nueva sociedad del conocimiento, ha dado lugar a un proceso cargado de burocracia y con objetivos que tras 20 años de funcionamiento aún distan mucho de haberse conseguido. En este sentido, los intentos encaminados a lograr cambios en las metodologías de enseñanza-aprendizaje; la gestión del personal docente e investigador; la estructura curricular o la movilidad estudiantil han sumado más críticos que adeptos dentro de la Comunidad Universitaria.

Con este marco de referencia, la Universidad española ha intentado ir adaptándose no sólo a las exigencias derivadas del mismo, sino también a otras, consecuencia directa de las nuevas normativas que se han ido aprobando e implementado en España en esos 20 años. Concretamente, en el caso que aquí nos interesa, aquellas derivadas de la Ley Orgánica 3/2007, de 22 de marzo, para la igualdad efectiva entre mujeres y hombres y de las modificaciones que afectaron a la Universidad, recogidas en la Ley Orgánica 4/2007, de 12 de abril que modificaba la Ley Orgánica 6/2001 de Universidades (LOU). E incluso, la Ley 14/2011 de Ciencia, Tecnología y la Innovación que dedica la disposición adicional $13^{\mathrm{a}}$ a la implantación de la perspectiva de género. Así como la Ley Orgánica 1/2004, de 28 de

\footnotetext{
6 Para un conocimiento detallado del contenido de la Declaración de Bolonia, véase: http://www.eees.es/pdf/Declaracion_Bolonia.pdf (25/07/2019).
} 
diciembre, de Medidas de Protección Integral contra la Violencia de Género ${ }^{7}$. En el ámbito andaluz destacan: (1) la Ley 12/2007, de 22 de noviembre, para la promoción de la igualdad de género en Andalucía, siendo uno de sus objetivos principales la eliminación de los estereotipos y las discriminaciones sexistas, (2) la Ley 13/2007, de 26 de noviembre, de medidas de prevención y protección integral contra la violencia de género ${ }^{8}$.

Hay que incidir en el Real Decreto 1393/2007, de 29 de octubre, por el que se establece la ordenación de las enseñanzas universitarias oficiales, donde se dispone la inclusión del principio de igualdad de oportunidades entre hombres y mujeres en la formación universitaria. Concretamente, en su artículo 3.5 plantea que este principio deberá incluirse en los planes de estudio en los que proceda. Así quedó recogido en el II Plan de Igualdad Efectiva entre Mujeres y Hombres de la Universidad de Jaén, concretamente en aquellas medidas que buscaban incidir en la oferta educativa de la mencionada Universidad. Concretamente, hay que mencionar el objetivo 2.1.1 (Incorporar docencia sobre la igualdad de género y no discriminación como elemento básico en la formación en valores del estudiantado) y 2.2.2 (proyectar una imagen libre de estereotipos por razón de género en las actividades docentes y en su documentación asociada).

Así, volviendo, a la conocida como Ley de Igualdad, suponía uno de los mayores logros del Gobierno del presidente José Luis Rodríguez Zapatero. Situando los temas de igualdad y equidad de género como uno de los pilares de su actividad política-gubernamental $\mathrm{y}$, en muchos sentidos la razón de ser de esa legislatura. Según palabras del citado Presidente, en el Congreso de los Diputados, el día de su aprobación (Congreso de los Diputados, 2007):

Para el Gobierno hoy es uno de los días más importantes de la legislatura, es también uno de los más felices. Hoy, aprobamos una de las normas más comprometidas con la igualdad de las que se conocen en el mundo. Hoy, el Parlamento español aprueba la Ley Orgánica para la igualdad efectiva de mujeres y hombres. Es una norma llamada a transformar, a transformar para bien, a transformar radicalmente y para siempre la sociedad española. Es una nueva norma de ampliación de derechos, de garantía de la efectividad de derechos. Hay quien ha dicho que, con la aprobación de dos leyes, la de promoción de la autonomía personal y de atención a las personas en situación de dependencia y esta que hoy votamos, la de igualdad entre mujeres y hombres, se justifica toda una legislatura.

Sin adentrarnos en otras cuestiones, los principios recogidos en dicha normativa deberían tener un reflejo directo y claro en el espacio universitario. Lo cual, conllevaba, en primer lugar, algunas modificaciones en la Ley Orgánica de Universidades para adaptarse a esta nueva realidad. En concreto, en relación a la formación en género, en su artículo 25, la Ley de Igualdad afirmaba que:

1. En el ámbito de la educación superior, las Administraciones públicas en el ejercicio de sus respectivas competencias fomentarán la enseñanza y la investigación sobre el significado y alcance de la igualdad entre mujeres y hombres;

\footnotetext{
7 En su articulo 4, punto 7, establece que “las Universidades incluirán y fomentarán en todos los ámbitos académicos la formación, docencia e investigación en igualdad de género y no discriminación de forma transversal”.

${ }^{8}$ En su artículo 16 establece como líneas de actuación en el ámbito de la enseñanza universitaria: (1) el fomento de estudios y conocimientos transversales para promover el desarrollo emocional, coeducación, prevención de violencia de género y las relaciones de igualdad, (2) promoción de contenidos sobre violencia de género que formen a profesionales de la enseñanza, y (3) promoción de contenidos sobre violencia de género en los estudios de grado y postgrado.
} 
2. En particular, y con tal finalidad, las Administraciones públicas promoverán: a) La inclusión, en los planes de estudio en que proceda, de enseñanzas en materia de igualdad entre mujeres y hombres. b) La creación de postgrados específicos. c) La realización de estudios e investigaciones especializadas en la materia.

Por tanto, como acabamos de afirmar, dicha normativa obligó a la modificación de la Ley de Universidades que hasta ese momento estaba funcionando en España y dio lugar a la aprobación de la Ley Orgánica 4/2007, de 12 de abril, de Universidades. En la misma se leía en su Exposición de Motivos que:

Esta Ley no olvida el papel de la Universidad como transmisor esencial de valores. El reto de la sociedad actual para alcanzar una sociedad tolerante e igualitaria, en la que se respeten los derechos y libertades fundamentales y de igualdad entre hombres y mujeres, debe alcanzar, sin duda, a la Universidad... Además, esta reforma introduce la creación de programas específicos sobre la igualdad de género.

Estos cambios, dieron lugar a un atisbo de ilusión entre aquellas personas que durante tanto tiempo venían reclamando un espacio en las Universidades, para lo que en otros países se conocían como “estudios de género", “estudios feministas” y/o “estudios de las mujeres”. Un área de conocimiento que permitiera introducir en la docencia - puesto que en la investigación muchas de ellas ya lo hacían aunque sin respaldo institucional - esa nueva o desconocida metodología que era la perspectiva de género. En palabras de la profesora Rebollo (2013, P. 4):

La inclusión de la perspectiva de género en educación propone incorporar este conjunto de saberes y prácticas que reivindican el derecho de las personas a ser iguales desde la diferencia, introduciendo una agenda educativa para promover la igualdad real entre hombres y mujeres que abarca no sólo contenidos o áreas de atención sino también estrategias o formas de acción propias.

No por menos, si existe un acuerdo acerca de cuál es el papel que la Universidad debe tener en el siglo XXI, este es su rol como "sujeto transformador de la realidad social en materia de igualdad de género" (Menéndez, 2018, p.3). Las cuestiones relativas a la equidad de género se han convertido en un eje prioritario en la educación superior puesto que una de sus funciones es formar una ciudadanía crítica que trabaje por la igualdad social. A través del conocimiento de cuáles son las raíces de las desigualdades que aún perviven entre mujeres y hombres se podrán buscar mecanismos que permitan construir democracias de calidad donde impere la defensa de la dignidad y los derechos humanos. Todo ello, en un momento donde luchar contra la desigualdad es, al mismo tiempo, luchar contra todos los tipos de violencia hacia las mujeres. En palabras de Donoso y Velasco (2013, p. 84) "la formación en género en educación superior se impone por varios motivos, entre los más importantes dotar al estudiantado de un marco interpretativo de la realidad que encaja con mayores elementos de justicia social y construye un mundo más equitativo y humano”.

\section{3. ¿DE QUÉ HABLAMOS CUANDO USAMOS EL CONCEPTO "PERSPECTIVA DE GÉNERO” EN LA DOCENCIA?}

Aunque para aquellas personas que llevan años trabajando con perspectiva de género la siguiente apreciación pueda parecer obvia, una docencia con perspectiva de género no significa realizarla sobre las mujeres (Agència per a la Qualitat del Sistema Universitari de Catalunya, 2019, p. 13) igual que no podemos entender género como sinónimo de sexo, ni de 
mujer ${ }^{9}$. En muchas ocasiones nos encontramos con que aquellas personas que trabajan, investigan o incluyen en sus programas docentes algún tema sobre la mujer, consideran que están trabajando con "perspectiva de género" y no siempre es así. Visibilizar a las mujeres en plural - dentro de los distintos temas que abarca una guía docente es un primer paso importante pero, no debe ser, sino el punto de partida para avanzar en el análisis y el conocimiento de las diversas aportaciones que en las distintas áreas de conocimiento han producido los hombres y las mujeres a lo largo de la historia y que nos han llevado a la situación actual. El impacto de esta perspectiva de análisis social implica "prestar atención a las similitudes y diferencias en las experiencias, los intereses, las expectativas, las actitudes y los comportamientos de las mujeres y de los hombres, así como identificar las causas y consecuencias de la desigualdad de género, para poder combatirlas (Agència per a la Qualitat del Sistema Universitari de Catalunya, 2019, p. 13). Es decir, a través de su utilización se "enriquece los procesos epistemológicos y otorga nuevas herramientas conceptuales y prácticas para la comprensión de diversas problemáticas sociales que se originan en la desigualdad entre hombres y mujeres” (Buquet, 2011, p. 214).

La perspectiva de género, por tanto, es un marco de análisis teórico y conceptual que permite (Pacheco, 2004, p.11):

- Visualizar la condición y posición de las mujeres con respecto a los hombres separando las diferencias sexuales de las representaciones sociales que se construyen cultural e históricamente (género) a partir de la diferencia sexual (sexo).

- Detectar los factores de desigualdad en los distintos ámbitos.

- Planificar acciones para modificar los elementos que mantienen las desigualdades, así como las relaciones asimétricas entre mujeres y hombres.

Biológicamente podríamos afirmar, con muchos matices, que no ha cambiado lo que son las mujeres y los hombres, pero sí se ha producido un cambio en cómo las sociedades definen "lo masculino" y "lo femenino" (Rodríguez y Provencio, 2017b). Lo cual guarda estrecha relación con los estereotipos de género y el uso que dentro de las ciencias sociales se hace de los mismos dependiendo de cuáles serían las características y los comportamientos que se considerarían "típicos" de los hombres y de las mujeres. Uno de los ejemplos más llamativos en la Universidad tiene que ver, aún hoy, con el tipo de estudios que preferentemente eligen mujeres y hombres. En este sentido, ellas, siguen decantándose por grados que tienden a relacionarse con los cuidados y/o "el estar para otros", tales como enfermería, magisterio, trabajo social y/o psicología (por citar algunos) y ellos, suelen hacerlo por las ingenierías, telecomunicaciones y/o grados de ciencias. Este estereotipo suele estar tan arraigado (a pesar de los esfuerzos institucionales por acabar con él ${ }^{10}$ ) que al final se convierte en la profecía autocumplida. En palabras de Marta Macho: "hay numerosos estudios que demuestran que

\footnotetext{
${ }^{9}$ El sexo es una condición biológica, genética y fisiología de nacimiento, hace referencia a la dicotomía Hombre-Mujer y es algo considerado "natural”. Mientras que el género está determinado socialmente, culturalmente y aprendido. Hace referencia a la dicotomía: Masculino-Femenino. Es algo considerado "social”. Para mayor información véase: Comisión Europea (2016): Guidance on Gender Equality in Horizon 2020. Recuperado de: https://eige.europa.eu/sites/default/files/h2020-hi-guide-gender_en.pdf (25/06/2019).

${ }^{10}$ Véase, entre otros, la Unidad de Mujeres y Ciencias del Ministerio de Ciencia, Innovación y Universidades. Recuperado. de: http://www.ciencia.gob.es/portal/site/MICINN/menuitem.26172fcf4eb029fa6ec7da6901432ea0/?vgnextoid=25c 55ef3677c4610VgnVCM1000001d04140aRCRD y el Programa For Women in Science, auspiciado por la Fundación L'Oréal y la Unesco cuyo fin último es “apoyar y reconocer a las mujeres investigadoras, para alentar a más mujeres jóvenes a ingresar a la profesión y acompañarlas una vez que sus carreras despegan”. Recuperado de https://www.forwomeninscience.com/es/home (17/07/2019)
} 
desde los seis años, debido al entorno social, las niñas van acumulando inseguridades para dedicarse a carreras STEM ${ }^{11 ” " ~(E l ~ P a i ́ s, ~ 7 / a g o s t o / 2018) . ~ E s t a ~ c o n s t a t a c i o ́ n, ~ j u n t o ~ c o n ~ l a ~ f a l t a ~ d e ~}$ referentes mujeres, frena a muchas jóvenes para elegir este tipo de estudios. Lo cual, además, está íntimamente relacionado con las expectativas del profesorado en relación a las acciones que se desarrollan en el aula según el sexo del estudiantado y al patrón del comportamiento que se espera de hombres y mujeres en los contextos de interacción del proceso enseñanzaaprendizaje y al de conducta dentro de las salas de clase ${ }^{12}$.

En el ámbito investigador y docente dentro de la universidad, el número de ellas a medida que se accede en la jerarquía a posiciones de alto nivel es menor. Mientras que las diferencias en número en las figuras de ayudante doctor y contratado son leves en las de catedrático son elevadas. Gallego y Matus (2018, p. 213) señalan que en 2015 existe un catedrático por cada 6,5 profesores y una catedrática por cada 15,4 profesoras. En cuanto a su presencia en la estructura organizativa universitaria, en el curso académico 2014/2015 las mujeres suponían solo el $2 \%$ en la dirección de la Universidad, y el 38,3 \% de los vicerrectorados (Díaz y Cuevas, 2015). Además de factores de índole personal o familiar y de carácter organizacional, Guil $(2005,2007)$ señala también los factores sociales como barreras para el acceso a categorías más altas. En estos factores encontraríamos, por un lado, los roles y estereotipos de género y, por otro lado, la visión androcéntrica sobre la base de una sociedad machista y patriarcal.

Por tanto:

Una docencia con perspectiva de género mejora la calidad docente y la relevancia social de los conocimientos, las tecnologías y las innovaciones producidas. La perspectiva de género permite profundizar en la comprensión de las necesidades, los comportamientos y las actitudes del conjunto de la población, rehuyendo así tanto las interpretaciones parciales que parten del hombre como sujeto universal como los esencialismos sobre los hombres y sobre las mujeres (Agència per a la Qualitat del Sistema Universitari de Catalunya, 2019, p. 14)

Además, estimula el pensamiento crítico del estudiantado y desarrolla competencias que les permitirán, cuando tengan que introducirse en el mercado laboral, superar lo que se ha denominado como la ceguera de género (gender blindness) ${ }^{13}$. Cuyas principales consecuencias son:

La falta de reconocimiento de que los roles y las responsabilidades de las mujeres/niñas y los hombres/niños se les atribuyen o se les imponen en contextos sociales, culturales, económicos y políticos específicos. Los proyectos, los programas, las políticas y las actitudes ciegas al género no tienen en cuenta estos roles diferentes y necesidades diversas y, por lo tanto, mantienen el statu quo y no ayudan a transformar la estructura desigual de las relaciones de género (EIGE, s/f).

\footnotetext{
${ }^{11}$ Ciencia, Tecnología, Ingeniería y Matemáticas por sus siglas en inglés. Según datos del Ministerio de Educación, sólo uno de cada cuatro matriculados en ingeniería es mujer. Y según el Instituto de la mujer, sólo el 31,4\% de las personas matriculadas en STEM son mujeres. El País (7/agosto/2018): “¿Por qué ellas no eligen carreras técnicas?”. Recuperado

de https://elpais.com/economia/2018/07/25/actualidad/1532514376_084338.html (17/07/2019).

${ }^{12}$ Para una mayor información sobre estos temas véase las guías y el material elaborado por la Red Universidad, Docencia, Género e Igualdad (Universidad de Alicante). Recuperado de: https://web.ua.es/es/unidadigualdad/proyectos/red-universidad-docencia-genero-e-igualdad.html (17/07/2019).

${ }^{13}$ Las Unidades de Igualdad que forman parte de las Universidades de la Xarxa Vives publicaron 11 guías para una docencia universitaria con perspectiva de género. Su objetivo es a través de los recursos que ofrecen eliminar la ceguera de género en la docencia, la investigación y la gestión universitaria. Para mayor información véase las publicaciones recogidas en el siguiente enlace: https:/www.vives.org/coleccio/guies-per-a-unadocencia-universitaria-amb-perspectiva-de-genere/ (18/07/2019).
} 
O, si nos centráramos en la investigación, se entendería como la no consideración de la dimensión de género como categoría significativa para el abordaje e interpretación de los problemas de investigación. La cual es debido, sobre todo, a la falta de formación y concienciación en temas de género. A veces, tras una supuesta neutralidad de género, lo que se esconde es ceguera de género (Caprile, 2012, p. 6).

\section{PAUTAS PARA INTRODUCIR LA PERSPECTIVA DE GÉNERO A PARTIR DE LAS ACCIONES DOCENTES IMPLEMENTADAS EN LA UNIVERSIDAD DE JAÉN}

El siguiente paso, por tanto, una vez aceptada la necesidad de introducir la perspectiva de género en la docencia universitaria, es ver de qué modo y a través de qué estrategias podremos hacerlo. Algunas, aquellas que tienen que ver con nuestra actividad diaria dentro de las aulas, es decir, las que determinan el proceso de enseñanza-aprendizaje, ya sea a través de las guías docentes, el uso del lenguaje no sexista e inclusivo, entre otras cuestiones, dependerá, sobre todo, de nuestras acciones y/o actitudes personales (por supuesto, también de nuestra formación y de las herramientas de las que dispongamos). Otras, aquellas que guardan estrecha relación con las políticas institucionales de cada Universidad, por ejemplo, los planes de igualdad o los protocolos de actuación ante el acoso sexual y/o sexista, van a venir determinadas por los equipos de gobierno. En palabras de Donoso, "se trata de engendrar las disciplinas, de crear pedagogía, de cuestionar las políticas universitarias y el sistema (...) Un currículum generizado propone la reconstrucción histórica de la experiencia de las mujeres y la construcción de nuevos conocimientos a partir de esa experiencia” (2018, p. 36). Es más, supone un cambio en la política universitaria, en la gestión de los recursos, de los presupuestos, de los puestos de trabajo, etcétera (Donoso, 2018).

Empezando por la Universidad como institución, es determinante que cuente, tal y como establece la normativa, con una Unidad de Igualdad. En el caso que nos centramos, la Universidad de Jaén, esta se creó en el año $2009^{14}$, desde el cual viene funcionando ininterrumpidamente. La misma, ha dado lugar a dos planes de Igualdad y la evaluación del primero (actualmente se está realizando la evaluación del segundo con vistas a la redacción del tercer plan). Entre sus objetivos, hay que mencionar:

- Velar para que se cumplan las leyes y normas emanadas de políticas de igualdad, correctoras del desequilibrio entre mujeres y hombres.

- Realizar estudios y diagnósticos de las desigualdades entre mujeres y hombres en todos los sectores de la comunidad universitaria.

- Elaborar y velar por el cumplimiento del Plan de Igualdad vigente.

- Promover campañas de sensibilización, formación e información para toda la comunidad universitaria.

\footnotetext{
${ }^{14}$ Son la consecuencia del mandato que se desprende de la Ley de igualdad para las administraciones públicas y que se recogió en la mencionada Ley Orgánica de Universidades del 2007. Son las encargadas de las funciones relacionadas con el principio de igualdad entre mujeres y hombres en el sentido más amplio de la palabra. Su labor es la realización de diagnósticos desde la perspectiva de género en cada universidad contemplando, entre otros factores, el análisis de los planes de estudios, la normativa actual vigente, actuaciones formativas, las medidas de conciliación existentes y la publicidad institucional. Son, además, las encargadas institucionalmente de crear e implementar los Planes de Igualdad en las Universidades y en los últimos años están trabajando para la aprobación de Protocolos contra el acoso sexual en la Educación superior y para la lucha contra la violencia de género. Sin olvidar, que suele ser el organismo que dentro de la institución vela por el cumplimiento del uso de un lenguaje no sexista e inclusivo. Para conocer la Unidad de Igualdad de la Universidad de Jaén, véase: https://www.ujaen.es/servicios/uigualdad/ (22/07/2019).
} 
- Prevenir y proteger posibles situaciones de acoso sexual y acoso sexista que pudieran sucederse en nuestra universidad.

- Fomentar la enseñanza e investigación acerca de la igualdad efectiva entre mujeres y hombres.

Como ya se mencionó al comienzo de este trabajo, el II Plan de Igualdad Efectiva entre Mujeres y Hombres de la Universidad de Jaén $(2016-2019)^{15}$ recogía en el Eje 2 sobre las misiones propias de la Universidad dos objetivos que guardan estrecha relación con la necesidad de introducir la perspectiva de género en la docencia, a saber:

- Objetivo 2.1.1: Incorporar docencia sobre la igualdad de género y no discriminación como elemento básico en la formación en valores del alumnado.

- Objetivo 2.2.2: proyectar una imagen libre de estereotipos por razón de género en las actividades docentes y en su documentación asociada.

De ahí que, conocer el diagnóstico que en su día se realizó para llevar a cabo del Primer Plan de Igualdad y la Evaluación del mismo como base para la elaboración del Segundo, nos permitirá tener, a su vez, elementos para analizar la equidad de género al interior de la propia institución Universitaria. Saber dónde están las mujeres en los distintos estamentos de la Universidad - ya sea en el personal docente e investigador y/o en los órganos de gobierno nos dará herramientas para impulsar "acciones, programas y políticas institucionales diseñadas específicamente para generar cambios positivos en las relaciones de género" (Buquet, 2011, p. 220).

El siguiente paso consistiría en analizar los distintos grados que se ofertan en la Universidad. En este caso, de los 50 que actualmente están a disposición del estudiantado no existe ninguno específico sobre igualdad. Aunque si se da un paso más, en postgrado, sí que existe un Máster oficial (entre los 52 ofertados para el curso 2019/20), concretamente el Máster Universitario en Análisis Crítico de las Desigualdades de Género e Intervención Integral en Violencia de Género, el cual además está dentro de la Facultad de Ciencias Sociales y Jurídicas.

En el tema que aquí nos interesa, en la citada facultad - la cual cuenta por vez primera con una mujer como decana- entre las materias que se imparten dentro de los distintos grados ofertados, no hay ninguna asignatura específica de género en ninguno de los planes aprobados en las últimas reformas ${ }^{16}$. Ello, a pesar de que la normativa de la ANECA obligaba en su "Protocolo de evaluación de Títulos Universitarios Oficiales de Grado y Máster" a que en los planes de Estudios en que procediera se incluyeran enseñanzas relacionadas con los derechos fundamentales y de igualdad de oportunidades entre hombres y mujeres (ANECA, 2011, p. 3). Así mismo, en la Facultad tampoco es posible encontrar asignaturas trasversales de género a los distintos grados. Lo que sí es posible encontrar, en algunos grados, son asignaturas que dentro de sus contenidos han incluido estos temas. Es aquí donde consideramos que se puede hacer un primer trabajo para la introducción de esta perspectiva de género, no por menos, como hemos venido afirmando a lo largo de este trabajo, "incorporar la perspectiva de género en los contenidos de una asignatura ofrece la posibilidad de introducir las herramientas conceptuales para que el estudiantado estudie y analice críticamente la parcela de la realidad objeto de presentación con criterios de interpretación sin sesgos de género" (Rodríguez y Provencio, 2017a, p.30). En palabras de la profesora Donoso, "es un desafío para el

\footnotetext{
${ }^{15}$ Tanto el Primer como el Segundo Plan de Igualdad de la Universidad de Jaén se pueden revisar en la página de la Unidad de Igualdad de citada Universidad. Así mismo, la Evaluación del primer plan. En la actualidad se está realizando la evaluación del II Plan que dará lugar al III Plan que se realizará en el 2020. https://www.ujaen.es/servicios/uigualdad/unidad-de-igualdad/planes-de-igualdad (26/07/2019).

${ }^{16}$ El análisis se realizó en el marco del proyecto de innovación docente PID10_201416.
} 
profesional el ahondar en cómo su disciplina se relaciona con los temas de género” (2018, p. 38) y ello porque la Universidad no puede seguir ajena a los temas de género ya que la trasformación que propugna redundaría en beneficio de toda la sociedad en su conjunto.

En este sentido, para poder seguir ahondando en qué se puede hacer para introducir la perspectiva de género en nuestra docencia, tendríamos que diferenciar entre las modificaciones que se podrían efectuar en los contenidos del currículum formal, es decir, en aquel que hace referencia a lo que aparece explícitamente recogido en las guías docentes de las asignaturas y en el de los contenidos del currículum oculto, el cual según la profesora Maceira serían (2005, p. 195):

Los aprendizajes (valores, actitudes, conocimientos, destrezas, significados, habilidades, supuestos) no explícitos y/o no intencionales que se dan en el contexto escolar, no sólo en el aula o en el proceso de enseñanza mismo, sino que reproducen y/o expresan tanto en elementos vinculados a este proceso (...) como en las relaciones de poder y con la autoridad, códigos y formas de comunicación, lenguaje verbal y no verbal, interacciones escolares y sociales, ambiente escolar y de trabajo, actividades extraescolares, discursos, normas y políticas institucionales, la organización y gestión de la institución/sistema escolar y, por supuesto, en los criterios y supuestos con los que se eligieron y conformaron todos estos componentes escolares.

En el primer paso, siguiendo el modelo expuesto por la Universidad de Alicante (Rodríguez y Provencio, 2017b) pero aplicado a la Universidad de Jaén, se tendrían que tener en cuenta los elementos que se enumeran a continuación, a saber ${ }^{17}$ :

1. Datos Básicos de la asignatura.

2. Datos Básicos del Profesorado.

3. Prerrequisitos, contexto y recomendaciones.

4. Competencias y Resultados de Aprendizaje.

5. Contenidos.

6. Metodología y Actividades.

7. Sistema de Evaluación.

8. Documentación /Bibliografía.

9. Cronograma.

Nos interesa centrarnos, sobre todo, en los puntos 5, 6, 7 y 8, pero sin olvidar el 2 y el 3 . El 2 porque es en este en donde se recoge, de manera explícita, el nombre completo del profesorado encargado de la materia y su cargo académico. Ello permitiría visualizar el espacio ocupado por las mujeres en las distintas asignaturas y su "peso" en la docencia impartida. Por otra parte, el apartado 3 nos posibilitaría hacer una llamada hacia la necesidad de usar un lenguaje no sexista e inclusivo tanto en la docencia como en la evaluación de estos contenidos - enfatizando la penalización en caso de no utilizarse - así como la introducción de que nos encontramos ante una guía elaborada conforme a las normativas vigentes y con enfoque de género ${ }^{18}$. En el caso del punto 5, que hace referencia a "los contenidos", aquí es

\footnotetext{
${ }^{17}$ En la bibliografía de este trabajo se pueden encontrar algunas de las guías y materiales que se han ido realizando por otras Universidades españolas para introducir la perspectiva de género en la docencia universitaria.

${ }^{18}$ En el II Plan de Igualdad de la Universidad de Jaén, se recogía la siguiente acción: 2. 1.2.1: Revisar las guías docentes y programas de las asignaturas impartidas en la Universidad de Jaén para asegurar su adecuación a las recomendaciones en materia de utilización no sexista del lenguaje. Para mayor información véase: https://www.ujaen.es/servicios/uigualdad/unidad-de-igualdad/planes-de-igualdad (26/07/2019).
} 
donde el profesorado tiene un mayor margen de maniobra, los cuales no se disponen si lo que analizamos son las competencias y/o los resultados de aprendizaje. En este sentido, esta libertad de cátedra (aún dentro de unos límites) permite, entre otras cuestiones, visibilizar el papel de las mujeres en las distintas ramas del conocimiento, reduciendo de este modo los sesgos de género y el androcentrismo que ha caracterizado la trasmisión del conocimiento. Todo ello, porque no podemos nunca olvidar que la práctica docente no es neutra al género ya que el profesorado está socializado en patrones de género diferentes. Ello puede llevar a seleccionar o priorizar algunos temas o problemas dentro de la amplitud de un programa académico, frente a otros.

En la guía de recomendaciones para la inclusión de la perspectiva de género en la docencia universitaria, de la Red Universidad, Docencia, Género e Igualdad, se afirma que en relación a los contenidos, se deberían contemplar los siguientes temas (Rodríguez y Provencio, 2017 , p.12), a saber:

- La distinción entre lo biológico de lo social y culturalmente aprendido (roles y estereotipos de género).

- La identificación de las características que específicamente define a las mujeres y hombres.

- La visibilización que la relación entre hombres y mujeres establece.

- La utilización del concepto de género como categoría de análisis para la descripción e interpretación de las relaciones entre mujeres y hombres considerando las construcciones sociales y culturales.

- La identificación de la desigual distribución de recursos que se produce entre mujeres y hombres.

- La diferenciación conceptual y operativa entre sexo (diferencias biológicas variable cuantitativa) y género (diferencias construidas socialmente-categoría de análisis explicativa).

- La desigualdad y discriminación histórica de las mujeres como colectivo.

- La visibilización de la aportación de las mujeres académicas y científicas en el asunto de interés.

- La visibilización de las desigualdades descubriendo las brechas de género en el asunto de interés.

Por su parte, en relación a la metodología y a las actividades que se llevan a cabo para realizar el proceso enseñanza-aprendizaje, tanto en el aula como fuera de ésta, existen distintos trabajos que llaman la atención sobre la necesidad de hacer un proceso de reflexión y selección de qué vamos a hacer y cómo lo vamos a hacer si lo que buscamos es que haya un menor sesgo de género en nuestra docencia. Es decir, la mayoría del profesorado no piensa en que la metodología que utiliza para transmitir los conocimientos de su asignatura y las actividades que implementan va a tener distintos efectos en mujeres y en hombres. En este sentido, algunos trabajos nos llaman la atención, por ejemplo, en cómo se hacen los grupos: cómo se selecciona al estudiantado que va a formar parte de los mismos; cuántas mujeres y hombres hay; quién suele asumir el papel de líder o portavoz; qué papel suelen desempeñar las alumnas en estos, etcétera. Así mismo, también es interesante reflexionar sobre cómo se asignan los temas de los trabajos y qué temas se asignan; en los usos del tiempo cuando realizamos debates en clase y/o presentaciones; entre otras cuestiones. 
Centrándonos en los sesgos, sería necesario tener en cuenta las siguientes herramientas para poder detectar los mismos a la hora de plantear estudios de casos, resolución de problemas o trabajos con proyectos, entre otras opciones (Rodríguez y Provencio, 2017a ${ }^{19}$ ):

- Presentar los datos desagregados por sexo con el fin de ofrecer descripciones y explicaciones diferenciadas según género.

- Analizar los datos/resultados considerando las relaciones y roles de género.

- Ofrecer conclusiones/resultados generales y específicos para hombres y mujeres.

- Evidenciar, si las hubieran, las brechas de género y los factores de desigualdad que puedan darse.

- Analizar la tendencia o evolución que el asunto objeto de interés refleja en las mujeres y los hombres.

- Eludir sesgos de género, roles de género tradicionales o prejuicios de género en los ejemplos y ejercicios y prácticas propuestas.

- Proponer ejemplos, ejercicios y prácticas que pongan de manifiesto las desigualdades entre mujeres y hombres. Estos ofrecen elementos para la deconstrucción de las formas de discriminación existentes y transmite valores de equidad y respeto.

- Cuando se ofrezca la opción de que el estudiantado elija el tema sobre el que versará la práctica, ejercicio, proyecto,... se deberán plantear distintas opciones que motiven y estimulen tanto a ellas como a ellos.

Por último, pero no por ello menos importante, tendríamos que considerar el tema de la evaluación, que es tal vez uno de los que supone mayores dificultades a la hora de encararlo. Los pocos estudios que se han hecho han demostrado que existen diferencias de género en función de la herramienta de evaluación que se utilice. En este sentido, "por ejemplo, se han detectado diferencias de género en los resultados del modelo de examen final en el aula y los del examen en casa (take-home exam), así como en los exámenes de tipo test en función del tiempo proporcionado y de si las respuestas incorrectas se penalizan o no" (Agència per a la Qualitat del Sistema Universitari de Catalunya, 2019, p. 15). Lo que en última instancia subyace en el planteamiento de la introducción de la perspectiva de género en la evaluación de la docencia es que la diversidad del estudiantado nos debería llevar a realizar distintas pruebas que permitieran contemplar las necesidades y especificidades del abanico de estudiantes que hay en cada grupo. Siendo conscientes de las dificultades que ello entraña tanto por el número de estudiantes que hay que evaluar, como con la carga burocrática y docente que el Espacio Europeo de Educación Superior y los criterios de la ANECA han supuesto para el día al día del profesorado ${ }^{20}$. Aun así, se debería hacer un esfuerzo por publicitar siempre los criterios de manera clara y con anterioridad a las pruebas escritas u orales; considerar las diferencias en cuanto al comportamiento comunicativo de mujeres y hombres y, en la medida en que sea posible, diseñar estrategias de evaluación que contemplen los procesos diferenciales de socialización en género.

No podemos terminar sin hacer mención a la bibliografía, que guardaría relación con los recursos docentes que utilizamos y que posteriormente mencionaremos al hablar del

\footnotetext{
${ }^{19}$ No hay que olvidar tampoco la importancia que tendrían estas cuestiones en la realización de Trabajos Fin de Grado y Trabajos Fin de Máster.

${ }^{20}$ Para mayor información véase las propuestas realizadas por la Universidad de Alicante en las distintas guías recogidas en su página web para la introducción de la perspectiva de género en la docencia. Red Universidad, Docencia, Género e Igualdad (Universidad de Alicante) Recuperado de: https://web.ua.es/es/unidadigualdad/proyectos/red-universidad-docencia-genero-e-igualdad.html (29/07/2019).
} 
currículum oculto. En este sentido, son conocidos los estudios que demuestran que se tiende a referenciar más autores que autoras, independientemente del área de conocimiento en que se esté trabajando. Además, cuando sólo se menciona en la bibliografía la inicial, se tienden a pensar que es un hombre y no una mujer quien ha realizado el artículo, el documento, etcétera. En este sentido, se aboga por incluir más mujeres en las bibliografías recomendadas; en los documentos que se trabajen en clase; en los artículos que se utilicen como fuente de referencia, etcétera. Hay que visibilizar el trabajo de las mujeres.

En relación al currículum oculto, hay que destacar, sobre todo, los siguientes aspectos:

- El uso del lenguaje no sexista y/o inclusivo

o En este caso, remitimos a la "Guía para un uso igualitario y no sexista del lenguaje y de la imagen en la Universidad de Jaén” recogido en la página web de la Unidad de Igualdad ${ }^{21}$.

- La interacción en el aula

o Para incluir la perspectiva de género tendremos que trabajar con los distintos estilos de comunicación y cómo podemos fomentar la mayor participación femenina en el aula.

- Los Recursos Docentes

o Nos lleva, por una parte, a realizar un autoanálisis de nuestros materiales, apuntes, libros, artículos propuestos, tanto escritos como visuales y audiovisuales. Y, por otra, a tener en cuenta dos aspectos interrelacionados, a saber:

- La comunicación oral en las clases: visualizar, nombrar, alternar y equilibrar.

- La comunicación escrita y gráfica en los apuntes, presentaciones, etcétera: visualizar, nombrar, alternar y equilibrar.

- Ejemplos utilizados en la docencia: diversidad y equidad.

\section{CONCLUSIONES}

A lo largo de las dos últimas décadas la normativa legal y la introducida para la reforma de los estudios universitarios en el marco de armonización del Espacio de Educación Superior en Europa ha reconocido y establecido la necesidad de incorporar la perspectiva de género en los títulos de grado y postgrado. Es cierto que tanto la normativa como autonómica y así como ciertas acciones desarrolladas en el contexto universitario ponen de manifiesto el interés por parte de la Administración educativa. Pero, a pesar de esto, y en el contexto de la Universidad de Jaén, asimilable a la universidad en España, aunque se visibiliza el interés en incorporar la perspectiva de género estamos lejos de materializarse en actuaciones concretas a nivel docente. La Universidad tiene que ir al frente de la sociedad, construir conocimiento, innovar en prácticas, asesorar gobiernos e instituciones. No podemos olvidar que la Universidad marca la vida de miles de personas. No puede perder la oportunidad de ayudar a que la vida de cada una de estas personas sea una caja de resonancia en pro de la justicia social y de género (Donoso 2018, p. 48).

A través de distintos proyectos de innovación docente, así como mediante cursos de formación del profesorado y prácticas docentes se ha ido implementando la perspectiva de

\footnotetext{
${ }^{21}$ Recuperado de: https://www.ujaen.es/servicios/uigualdad/sites/servicio_uigualdad/files/uploads/Guia_lenguaje_no_sexista.pdf $(29 / 07 / 2019)$
} 
género en la docencia universitaria en la Universidad de Jaén. Este trabajo introduce una serie de pautas para incluir la perspectiva de género en la docencia universitaria derivadas de las experiencias analizadas. El enfoque supone una restructuración del diseño del proceso de enseñanza-aprendizaje atendiendo a cuatro elementos: contenidos, metodología y actividades, sistemas de evaluación y bibliografía. Esto supone adoptar un enfoque con un carácter más transversal que no dependa de la voluntariedad del profesorado y su implicación personal y profesional con estas temáticas, sino en el compromiso institucional con la igualdad y con la necesidad de trabajar con perspectiva de género en todas sus instancias. El personal docente e investigador que ya está sensibilizado, seguirá formándose y capacitándose a lo largo de toda su carrera profesional, muchas veces, incluso a costa de la penalización que supone en algunos momentos para su carrera profesional y/o del hándicap que conlleva la identificación con ciertas etiquetas dentro de la Universidad, por ejemplo, el feminismo. Pero, entendemos que como lo que planteamos no puede ser analizado como un tema "simbólico" o de segundo orden, sino prioritario para la consecución de los parámetros e indicadores de calidad derivados de compromisos y normativas internacionales, nacionales y autonómicas, también hay que, en cierta medida, convertirlo en un tema de obligado cumplimiento para toda la Comunidad Universitaria. De ahí la necesidad de asegurarse la consecución de las acciones recogidas en los Planes de Igualdad de las Universidades.

En resumen, aplicada a la docencia, la perspectiva de género supone un proceso de reflexión que afecta al diseño de las competencias del plan de estudios y, por extensión, al diseño de las asignaturas, incluyendo los resultados de aprendizaje, los contenidos impartidos, los ejemplos, el lenguaje utilizado, las fuentes seleccionadas, el método de evaluación y la gestión del entorno de aprendizaje. Para acompañar con éxito el proceso de incorporación de la perspectiva de género, resulta necesario que el personal docente adquiera esta competencia a través de la formación continua que ofrecen las unidades de innovación docente de las universidades y de las actividades formativas puntuales que organizan las unidades o los observatorios de igualdad (Agència per a la Qualitat del Sistema Universitari de Catalunya, 2019, p.18). Se ha trabajado en que dichos cursos se ofertaran, de igual modo, hay que seguir trabajando para que los mismos no queden en algo sin importancia sino que se tengan que realizar. Tal vez, su posible valoración positiva en la bolsa de trabajo o en los concursos para el ingreso en determinados cuerpos docentes, fueran un aliciente para aquella parte del profesorado que sigue pensando que trabajar con perspectiva de género suma tanto para su docencia como para quien la recibe.

En este sentido, considerando la importancia que para el estudiantado universitario tiene adquirir formación con perspectiva de género, podemos afirmar que impartir docencia bajo esta metodología no puede ser sólo valorado como un complemento deseable en la formación de los y las egresados, sino como la base de una formación en valores que mejore su formación universitaria y los/as capacite de un elemento diferenciador.

\section{BIBLIOGRAFÍA}

Agència per a la Qualitat del Sistema Universitari de Catalunya (2019): Marco General para la incorporación de la perspectiva de género en la docencia universitaria. Recuperado de: http://www.aqu.cat/doc/doc_25276332_1.pdf.

Agencia Nacional de Evaluación de la Calidad y Acreditación (2015): Guía de apoyo para la elaboración de la Memoria para la solicitud de verificación de títulos oficiales. Recuperado de: http://www.aneca.es/Programas-de-evaluacion/Evaluacion-detitulos/VERIFICA/Verificacion-de-Grado-y-Master/Documentacion-y-herramientas. 
ANECA (2011): Protocolo de evaluación para la verificación de títulos universitarios oficiales (grado y máster). Recuperado de: http://www.aneca.es/Programas-deevaluacion/Evaluacion-de-titulos/VERIFICA/Verificacion-de-Grado-yMaster/Documentacion-y-herramientas

Ayuntamiento de Barcelona (s/f): Para construir un mundo más igualitario. Guía de comunicación inclusiva. Recuperado de: https://ajuntament.barcelona.cat/guiacomunicacio-inclusiva/pdf/guiaInclusiva-es.pdf.

Bartual Figueras, M. T; Carbonell Esteller, M; Carreras Marín, A; Colomé Ferrer, J y Turmo Garuz, J. (2018): "La perspectiva de género en la docencia universitaria de Economía e Historia”, Revista d'Innovació Docent Universitària, nº 10, pp. 92-101. Recuperado de: http://revistes.ub.edu/index.php/RIDU

Blázquez Vilaplana, B. (2015): “Trabajar con perspectiva de género en la Ciencia Política: algunos apuntes sobre el caso de la Universidad española”, Revista Diálogo Possiveis, Salvador de Bahía (Brasil), Año 14, nº 2, pp. 71-91, Julio-Diciembre.

Blázquez Vilaplana, B. (2016): “Aproximación a la formación en género en el Espacio Europeo de Educación Superior a través del análisis de la Memoria y las guías docentes del Grado de Relaciones Laborales y Recursos Humanos en la Universidad de Jaén”, Internacional Journal of Educational Research and Innovation (IJERI), ${ }^{\circ} 5$, pp. 28-41.

Blázquez Vilaplana, B; Alarcón González, F. J. y Vela Díaz, R. (2018): “Las competencias y la igualdad de oportunidades en el grado universitario: una propuesta de análisis a partir de un estudio de caso”. En Rebollo Catalán, $\mathrm{M}^{\mathrm{a}}$ Ángeles: La Universidad en clave de género, Octaedro Editorial, Colección Universidad, Barcelona, pp. 53-68.

Buquet Colerto, A. G. (2011): "Transversalización de la perspectiva de género en la educación superior”. Perfiles educativos, Vol. XXXIII, No especial, pp. 213-225. Recuperado de: http://www.scielo.org.mx/pdf/peredu/v33nspe/v33nspea18.pd.

Caprile, M. (coord.) (2012): Guía práctica para la inclusión de la perspectiva de género en los contenidos de la investigación, CIREM Fundación, Madrid.

Comisión Europea (2016).Guidance on Gender Equality in Horizon 2020. Recuperado de: https://eige.europa.eu/sites/default/files/h2020-hi-guide-gender_en.pdf

Congreso de los Diputados (2007): Intervención del Presidente del Gobierno tras la aprobación de la Ley Orgánica para la Igualdad Efectiva entre Mujeres y Hombres. 15 de marzo de 2007. Recuperado de: http://www.iceta.org/zp150307.pdf

Díaz, F. y Cuevas, M. (2015): “Género y liderazgo en la universidad española. Un estudio sobre la brecha de género en la gestión universitaria”, Archivos Analíticos de Políticas Educativas, vol. 23, n 106 , pp.1- 26.

Donoso Vázquez, T. y Velasco Martínez, A. (2012): “La formación universitaria en género como toma de conciencia de las dinámicas generalizadas”. En Martín Clavijo, Milagros (edt): Más igualdad, redes para la igualdad, Arcibel Editores, pp 239- 248.

Donoso Vázquez, T y Velasco Martínez, A. (2013): “¿Por qué una propuesta de formación en perspectiva de género en el ámbito universitario?”, Profesorado. Revista de currículum y formación del profesorado, vol. 17, n 1, pp. 71-88.

Donoso Vázquez, T. (2018): Perspectiva de género en la Universidad como motor de innovación. En Rebollo Catalán, $\mathrm{M}^{\mathrm{a}}$ Ángeles: La Universidad en clave de género. Octaedro Editoria, Colección Universidad, Barcelona, pp. 23-52.

EGERA (s/f). Effective Gender Equality in Research and the Academia. Recuperado de: https://www.egera.eu/. Documento completo Recuperado de: https://www.uab.cat/doc/Good_practices_Gender_curricula.pdf.

El País (7/agosto/2018): “¿Por qué ellas no eligen carreras técnicas?”. Recuperado de https://elpais.com/economia/2018/07/25/actualidad/1532514376_084338.html. 
European Institute for Gender Equality (EIGE) (S/F): Glosario: Ceguera de Género. Recuperado de https://eige.europa.eu/thesaurus/terms/1157?lang=es.

Evaluación del Primer Plan de Igualdad de la Universidad de Jaén. Recuperado de: https://www.ujaen.es/servicios/uigualdad/sites/servicio_uigualdad/files/uploads/iguald ad/Documentos/PlanesIgualdad/EVALUACION\%20PLAN\%20IGUALDAD_B.pdf

Gallego-Morón, N. y Matus-López, M. (2018): “Techo de cristal en las universidades españolas. Diagnóstico y Causas”, Profesorado, Revista de Currículum y Formación del Profesorado, vol. 22, n 3, pp. 209-229.

Guerrero Salazar, S. (2012): Guía para un uso igualitario y no sexista del lenguaje y de la imagen en la Universidad de Jaén. Unidad de Igualdad de la Universidad de Jaén. Jaén. Recuperado de: https://www.ujaen.es/servicios/uigualdad/sites/servicio_uigualdad/files/uploads/Guia_l enguaje_no_sexista.pdf.

Guil, A. (2005): Techos de cristal en la Universidad de Sevilla, Ministerio de Trabajo y Asuntos Sociales, Madrid.

Guil, A. (2007): "Docentes e investigadoras en la universidades españolas: visibilizando techos de cristal”. Revista de Investigación Educativa, vol. 25, núm. 1, pp. 111-132.

Ley 39/1999, de 5 de noviembre, para promover la conciliación de la vida familiar y laboral de las personas trabajadoras. BOE $n^{0}$ 266. Recuperado de: https://www.boe.es/eli/es/1/1999/11/05/39.

Ley 12/2007, de 22 de noviembre, para la promoción de la igualdad de género en Andalucía. Boletín Oficial de la Junta de Andalucía no 247 de 18/12/2007. Recuperado de: https://www.juntadeandalucia.es/boja/2007/247/1.

Ley 14/2011, de 1 de junio, de la Ciencia, la Tecnología y la Innovación. Boletín Oficial del Estado $\quad n^{\circ} \quad 131$ de 02/06/2011. Recuperado de: https://www.boe.es/buscar/act.php?id=BOE-A-2011-9617.

Ley 13/2007, de 26 de noviembre, de medidas de prevención y protección integral contra la violencia de género. Boletín Oficial de la Junta de Andalucía número 247 de 18/12/2007. Recuperado de: https://www.juntadeandalucia.es/boja/2007/247/2.

Ley Orgánica 1/2004, de 28 de diciembre, de Medidas de Protección Integral contra la Violencia de Género. Boletín oficial del Estado n 313 de 29/12/2004. Recuperado de: https://www.boe.es/buscar/act.php?id=BOE-A-2004-21760.

Ley Orgánica 3/2007, de 22 de marzo, para la igualdad efectiva entre mujeres y hombres. Boletín oficial del Estado $\mathrm{n}^{0} 71$ Recuperado de: https://www.boe.es/eli/es/lo/2007/03/22/3/con.

Ley Orgánica 4/2007, de 12 de abril que modifica la Ley Orgánica 6/2001 de Universidades. Boletín oficial del Estado $\mathrm{n}^{\mathrm{0}}$ 89. Recuperado de: https://www.boe.es/eli/es/lo/2007/04/12/4.

Lopez-Frances, I. y Vazquez-Verdera, V. (2014): "La perspectiva de género y el papel de la universidad en el siglo XXI”. Teoría de la Educación. Educación, Cultura y Sociedad de la Información, vol. 15, nº 4, pp. 241-261.

Luengo Rodríguez, T. y Rodríguez Sumaza, C. (2009): “Enfoque de género en la docencia universitaria: apuntes para la elaboración de un protocolo de buenas prácticas”. En Ma Elena Jaime de Pablos (ed.) Identidades femenina en un mundo plural. AUDEM, pp. 441-447. Recuperado de: http://www.audem.com/publicaciones.php.

Maceira Ochoa, L. (2005): "Investigación del currículum oculto en la educación superior: alternativa para superar el sexismo en la escuela”. Revista de Estudios de Género. La Ventana, Universidad de Guadalajara, México, n 21, pp. 187-227. Recuperado de: http://www.redalyc.org/pdf/884/88402107.pdf. 
Martínez Moscoso, D. M. (2012): Práctica docente con equidad de género. Una guía de trabajo, Universidad de Guadalajara, Centro de Estudios de Género, Unidad de Igualdad, México.

Menéndez Baena, A.B. (2018): Análisis de la incorporación de la perspectiva de género en los Planes de Estudios de Grado en la Universidad de Cádiz, Universidad de Cádiz, Cádiz. Recuperado de: https://igualdad.uca.es/wpcontent/uploads/2018/09/An\%C3\%A1lisis-PG-UCA-2-copia.pdf?u.

Pacheco, C. (2004): Prácticas sexistas en el aula, UNICEF, Paraguay. Recuperado de: http://www.unicef.org/paraguay/spanish/py_practicas_sexistas.pdf.

Ponferrada, M. (2017): Guia per a la introducció de la perspectiva de génere en la docéncia. Tercer Pla d'acció per la Igualtat entre dones i homes de la UAB (2014-2017). Observatori per a la Igualtat, Universidad Autónoma de Barcelona, Barcelona. Recuperado de: https://www.uab.cat/doc/Guia_perspectivagenere_docencia.

Primer Plan de Igualdad de la Universidad de Jaén (2011-2015), Unidad de Igualdad, Universidad de Jaén, Jaén. Recuperado de: https://www.ujaen.es/servicios/uigualdad/sites/servicio_uigualdad/files/uploads/iguald ad/Documentos/PlanesIgualdad/PLAN\%20IGUALDAD\%20UJAEN\%2020112015.pdf.

Programa For Women in Science. Recuperado de https://www.forwomeninscience.com/es/home.

Real Decreto 1393/2007, de 29 de octubre, por el que se establece la ordenación de las enseñanzas universitarias oficiales. BOE $\mathrm{n}^{\circ}$ 260. Recuperado de: https://www.boe.es/buscar/pdf/2007/BOE-A-2007-18770-consolidado.pdf.

Rebollar Sánchez, E. M. (2013): El género en los Planes de Estudios de los Grados de Educación en las Universidades Públicas Españolas, Tesis doctoral, Universidad Autónoma de Barcelona, Barcelona. Recuperado de: https://www.tesisenred.net/bitstream/handle/10803/129102/emrs1de1.pdf?sequence=1.

Rebollo Catalán, Ma A. Ruiz Pinto, E. y Vega Caro, L. (2018): "Presentación: Balada triste de trompeta o el sueño de una igualdad que no llega” en Rebollo Catalán, $\mathrm{M}^{\mathrm{a}}$ Ángeles: $L a$ Universidad en clave de género, Octaedro Editorial, Colección Universidad, Barcelona, pp. 17-22.

Rebollo, M. A., Piedra, J., Sala, A., Sabuco, A., Saavedra, J. y Bascón, M. J. (2012): "La equidad de género en educación: Análisis y descripción de buenas prácticas en educación”. Revista de Educación. Ministerio de Educación, nº 358, pp. 129-152.

Rebollo Catalán, M ${ }^{\mathrm{a}}$ A. (2013): "Editorial: La innovación educativa con perspectiva de género. Retos y desafíos para el profesorado”. Profesorado. Revista de currículum y formación del profesorado, vol. 17, $\mathrm{n}^{\circ}$ 1, Monográfico, pp 3-6.

Red Universidad, Docencia, Género e Igualdad (Universidad de Alicante) Recuperado de: https://web.ua.es/es/unidad-igualdad/proyectos/red-universidad-docencia-genero-eigualdad.html.

Rodríguez Jaume, Ma J. y Provencio Garrigós, H. (coord.) (2017a): Guía de recomendaciones para la inclusión de la perspectiva de género en la docencia universitaria práctica (I), Red Universidad, Docencia, Género e Igualdad, Universidad de Alicante, Alicante. Recuperado de: http://rua.ua.es/dspace/handle/10045/72075.

Rodríguez Jaume, M ${ }^{\mathrm{a}}$ J. y Provencio Garrigós, H. (coord.) (2017b): Guía de recomendaciones para la inclusión de la perspectiva de género en la docencia universitaria práctica (II), Red Universidad, Docencia, Género e Igualdad, Universidad de Alicante, Alicante. Recuperado de: http://rua.ua.es/dspace/handle/10045/72075.

Segundo Plan de Igualdad Efectiva entre Mujeres y Hombres de la Universidad de Jaén (2016-2019), Universidad de Jaén, Jaén. Recuperado de: 
Blázquez Vilaplana, B., Alarcón González, J.

https://www.ujaen.es/servicios/uigualdad/sites/servicio_uigualdad/files/uploads/II\%20 PLAN\%20IGUALDAD\%20EFECTIVA\%20ENTRE\%20MUJERES\%20Y\%20HOM BRES\%20.pdf.

Unidad de Igualdad de la Universidad de Jaén (s/f): https://www.ujaen.es/servicios/uigualdad/.

Unidad de Igualdad de la Universidad de Vigo (2014): Guía de apoio para introducir a perspectiva de xénero na doencia da universidad de Vigo. Recuperado de: https://www.uvigo.gal/opencms/export/sites/uvigo/sites/default/uvigo/DOCUMENTO S/igualdade/Guxa_para_a_introducixn_da_perspectiva_de_xxnero_na_docencia.pdf.

Unidad de Mujeres y Ciencias del Ministerio de Ciencia, Innovación y Universidades. Recuperado de: http://www.ciencia.gob.es/portal/site/MICINN/menuitem.26172fcf4eb029fa6ec7da69 01432ea0/?vgnextoid=25c55ef3677c4610VgnVCM1000001d04140aRCRD.

Unión Europea (s/f): Declaración de Bolonia. Recuperado de: http://www.eees.es/pdf/Declaracion_Bolonia.pdf.

Ventura Franch, A. (2008): "Normativa sobre estudios de género y universidad”, Feminismos $\mathrm{n}^{\circ} 12$, pp. 155-184.

Verge Mestre, T. y Cabruja Ubach, T (2017): La perspectiva de génere en docéncia i recerca a les universitats de la xarxa vives. Situació actual i reptes del futur. Xarxa Vives d'universitats. Recuperado de: http://www.vives.org/PU3.pdf.

Xarxa Vives (2017): Guies per a una docència universitària amb perspectiva de gènere. Recuperado de https://www.vives.org/coleccio/guies-per-a-una-docencia-universitariaamb-perspectiva-de-genere/. 